cells with vacuolated cytoplasm were noted. Immunohistochemistry performed on tumour showed positive staining with calretinin, WT1, CAM5.2 and negative staining with Berep4, CD34, and S100.

Conclusion: Adenomatoid tumours mimicking testicular tumour can present a challenge for urologist to decide on the most appropriate surgery. Clinical awareness of the unusual location of adenomatoid tumour could elect patient for testis sparing surgery.

\section{EPITHELIAL SPARC EXPRESSION IS CORRELATED WITH POOR SURVIVAL AS WELL AS MMP-2 EXPRESSION OF INVASIVE BREAST CARCINOMA}

Ji Shin Lee, Ga-Eon Kim, Jae Hyuk Lee, Jong Hee Nam, Chan Choi

Department of Pathology, Chonnam National University Medical School, Gwangju, Republic of Korea

Background: Invasion and metastasis are the direct causes of mortality in breast cancer patients. Secreted protein acidic and rich in cysteine (SPARC) plays a crucial role in the process of tumor invasion and metastasis in some tumors. Matrix metalloproteinases (MMPs) degrade the extracellular matrix and participate in tumor invasion and metastasis.

Aims: The aims were to assess the role of SPARC alteration in the breast carcinogenesis and its association with outcome variables and MMP expression in invasive breast carcinoma (IBC). Methods: Immunohistochemical staining with anti-SPARC antibody was performed in a total of 300 patients with 26 normal breasts, 76 ductal carcinoma in situ (DCIS), and 198 IBC using tissue microarray. In addition, we also performed immunohistochemical staining for MMP-2 and MMP-9 in IBC.

Results: Epithelial SPARC expression appeared to increase progressively along the continuum of neoplastic changes from normal breast epithelium to IBC $(\mathrm{P}<0.001)$. The patients with high epithelial SPARC expression had a significantly poorer prognosis for disease-free and overall survival than those with low epithelial expression ( $p=0.002$ and $p=0.048$, respectively). Epithelial SPARC expression was independently associated with an increased risk for poor disease-free survival. Epithelial SPARC expression was statistically correlated with MMP-2 expression $(p<0.05)$.

Conclusions: Tumor progression in breast epithelium is accompanied by increased epithelial SPARC expression. High epithelial SPARC expression may serve as a new parameter for the prognostic prediction in patients with IBC. SPARC-mediated degradation of the extracellular matrix, and its possible association with MMP-2, may contribute to the progression of IBC.

\section{HEPATIC REACTIVE LYMPHOID HYPERPLASIA ASSOCIATED WITH MALIGNANT TUMOR}

Sik Lee, Jun Sang Bae, Woo Sung Moon

Department of Pathology, Internal Medicine, Chonbuk National University, Medical School, and Research Institute for

Endocrine Sciences, Jeonju, Korea
Reactive lymphoid hyperplasia (RLH) is a rare benign condition that forms a mass-like lesion characterized by proliferation of non-neoplastic lymphocytes forming follicles and germinal centers. RLH is thought to represent a reactive immunological response, and some cases may arise in association with malignancy. Herein, we present four cases of hepatic RLH associated with malignant tumor clinically mimic metastatic tumor. All 4 patients were women ranging from 45 to 74 years (mean, 59 years). The hepatic lesions ranged from 0.5 to $1.5 \mathrm{~cm}$. Radiologically, these lesions mimic a primary or metastatic malignancy. All 4 lesions were associated with internal malignancies; renal cell carcinoma, ovarian mucinous carcinoma, colon adenocarcinoma and bile duct carcinoma, respectively. Microscopically, the lesions are characterized by formation of follicles with polymorphic and polyclonal cell population and active germinal centers. There was no evidence of lymphoepithelial lesion or monoclonal B-cell proliferation. It can be suggested that the pathogenesis of RLH of the liver in patients with malignant tumor may be related to an immunologic abnormality that is caused by the malignant tumor itself or previous surgery for the tumors.

\section{SELECTION OF SUPER-INVASIVE CELL POPULATION FROM THE GLIOBLASTOMA CELL LINE AND ANALYSIS OF INVASION-ASSOCIATED BIOMARKERS}

Kyu Sang Lee, Hyun Sook Jung, Seyoung Moon, Gheeyoung Choe

Department of Pathology, Seoul National University Bundang Hospital, Seoul National University College of Medicine, Korea

Background: Glioblastoma is characterized by aggressive local invasiveness, making complete surgical resection nearly impossible. To identify mechanisms of glioblastoma invasion, we isolated a subpopulation of more invasive cells and evaluated the expression of invasion related factors including integrin subunits, matrix metalloproteinase-2 (MMP2), fascin, actin and Erk1/2.

Methods: We selected the first $10 \%$ of invading cells (U87-Inv) from U87MG using laminin-2 (merosin) coated Transwell filters. To characterize the more invasive cells, we used wound assay, gelatin zymography, proliferation assay, RT-PCR for integrinreceptors for merosin, western blot and immunofluorescence for fascin and actin.

Results: Migration rate of U87-Inv cells exhibited about $20 \%$ increase compared with relatively lower invasive cells (U87Non). U87-Inv cells demonstrated faster wound healing, whereas lower proliferative activity. The expression levels of integrin $\alpha 1$, $\alpha 7$, and $\beta 1$ increased a little higher (about 1.2-fold for $\beta 1$ and 1.5 -fold for $\alpha 1$ and $\alpha 7$ ) in U87-Inv compared with U87-Non, while the expression of integrin $\alpha 6$ and $\beta 4$ reduced in U87-Inv (about 0.4 -fold for $\beta 4$ and 0.6-fold for $\alpha 6$ ). U87-Inv cells revealed increase of fascin, actin and MMP2, whereas they showed decrease of phosphorylated Erk1/2. By immunofluorescence study, U87-Inv cells revealed extensive lamellipodia expressing both fascin and actin. 